Gut, 1979, 20, 575-580

\title{
Value of sigmoidoscopy and biopsy in detection of carcinoma and premalignant change in ulcerative colitis
}

\author{
R. H. RIDDELL ${ }^{1}$ AND B. C. MORSON \\ From the Department of Pathology, St. Marks Hospital, London
}

SUMMARY Of 111 carcinomas developing in 73 patients with ulcerative colitis, $46(41 \cdot 5 \%)$ arose in the rectum where they are directly accessible to sigmoidoscopy. Fifty-eight per cent of single carcinomas developed in the rectum. The extent and frequency of rectal dysplasia was assessed by examining slides of rectal mucosa with an eyepiece micrometer. Slides from 46 patients with carcinoma and 22 patients with dysplasia but no carcinoma in whom proctectomy or proctocolectomy had been carried out were examined by this method. Thirteen of 15 patients with carcinoma of the colon $(87 \%)$ and $21 / 22$ patients $(95 \%)$ with large bowel dysplasia showed evidence only of rectal dysplasia. However, there was marked variability in the proportion of dysplastic rectal mucosa even in those patients with rectal carcinoma, while in some patients dysplasia was limited to a small focus. Because of the possibility of false negative biopsies due to sampling error, multiple biopsies should be taken to detect dysplasia. Their state should be recorded and deliberately varied at subsequent visits. Careful sigmoidoscopy and multiple biopsies in this study had potential value as an aid in the detection of $85-90 \%$ of all carcinomas. In practice the figure would almost certainly be lower due to intrinsic bias (see discussion) so that, although regular sigmoidoscopy and biopsy would be of great value when colonoscopy is not available, the latter should be included in any long-term programme of carcinoma prevention.

The prevention of carcinoma complicating ulcerative colitis depends on the identification and close follow-up of that subgroup of colitic patients known to be particularly predisposed to the development of carcinoma. These are patients with extensive or total colitis, particularly if the age of onset was before 25 years of age, and with a long history of disease, traditionally 10 years, despite the fact that $20 \%$ of all colitic cancers occur before this time (Mottet, 1971). Follow-up of this high risk group has created problems of management, which varies from proctocolectomy when total disease has been present for 10 years, through varying degrees of surveillance, to the taking of no particular precaution in view of the relative rarity of carcinoma as a complication of ulcerative colitis. Mottet (1971), combining several series, concluded that approximately $3 \cdot 0-3 \cdot 5 \%$ of all colitics will eventually

${ }^{1}$ Present address: Department of Pathology, University of Chicago, 950 East 59th Street, Chicago, Illinois 60637, USA.

Accepted for publication 13 February 1979 develop carcinoma. However, only about a fifth of all colitics have total large bowel involvement, and carcinoma will develop almost exclusively in this group. It would therefore be expected that patients in the high risk group have about a $15-18 \%$ chance of developing carcinoma, a figure similar to the $12.5 \%$ at 20 years suggested by Edwards and Truelove (1964).

If these figures are valid, it would seem inappropriate to sacrifice the entire large bowel of all patients in the high risk group just because of the risk of carcinoma, when at least $80 \%$ of them will probably never develop this complication. Conversely, the remainder who will develop carcinoma is too large a group to ignore.

A method is therefore required which will select patients in this high risk group who are particularly predisposed to develop carcinoma, or who have only very early invasive lesions potentially curable by local excision. To this end, clinical history, physical examination including sigmoidoscopy and biopsy, double contrast barium enema, and colonscopy can all be used. However, some of these are of 
use only in detecting relatively advanced tumours, while the efficacy of others remain to be determined.

In 1967, Morson and Pang described 'precancerous changes' in 12/134 specimens resected for ulcerative colitis $(9 \%)$. These were frequently extensive and involved the mucosa away from the tumours as well as in its immediate vicinity and often involved the rectum. In nine patients a report of premalignant change on rectal biopsy was to some extent responsible for subsequent proctocolectomy. In five of these patients an incidental carcinoma was found in the resested specimen. They concluded that regular rectal biopsy might be used to predict which patients were most likely to develop carcinoma even though this might be elsewhere in the colon.

The presence of these changes was confirmed in 1972 by Evans and Pollock who found widespread dysplasia in two of four patients with carcinoma, and by Hulten et al. (1972), who found premalignant changes in 22/25 patients with colitis and cancer. Subsequently, at the same hospital Myrvold et al. (1974), subjected 47 patients with ulcerative colitis to proctocolectomy after three to four rectal biopsies had been taken. Premalignant changes were found in seven of these, and in five a carcinoma was found in the resected specimen, being unsuspected in four. Other studies (Lennard-Jones et al., 1974; Yardley and Keren, 1974; Cook and Goligher, 1975; Issacson, 1976; Gewertz et al., 1976; LennardJones et al., 1977) have confirmed that premalignant changes are not always accompanied by carcinoma, although most carcinomas are accompanied by precancer which is frequently rectal. Several of these studies have also suggested that rectal precancer may not always be present or may be patchy.

The object of the present study was to assess the potential value of sigmoidoscopy and biopsy in the detection of carcinoma and premalignant change in both patients with colitis and carcinoma, and in patients with ulcerative colitis and dysplasia who had not developed carcinoma.

The incidence of carcinomas occurring in the rectum in patients with ulcerative colitis is important, as these should be detectable early in the course of their evolution by careful sigmoidoscopy and confirmed biopsy. The frequency and extent of dysplasia is important, for, if rectal biopsy is to be used for identifying which patients in the high risk clinical group should be subject to proctocolectomy, it is imperative to know if the rectum is always involved when dysplasia is present, and, if not, how frequently rectal dysplasia is present. It is also important to know whether rectal involvement by dysplasia occurs as a field change involving the entire mucosa or whether it is focal, for the latter would raise problems of sampling error.

\section{Method}

The site of carcinoma in ulcerative colitis was determined by examinations of the records or pathology specimens or slides of patients seen at, or referred to St. Marks Hospital, London, until the end of 1974. These were recorded by sex, site, and whether single or multiple.

The frequency and distribution of rectal dysplasia was carried out by examining histological sections of the rectal mucosa of 63 patients in whom proctectomy or proctocolectomy had been carried out, and who had unequivocal ulcerative colitis and either carcinoma (41) or dysplasia (22). Only mucosa that was unequivocally dysplastic was measured, and criteria used were a modification (Riddell, 1977) of those used by Morson and Pang. Conventional sections were up to one inch in length, but in many patients 'Swiss roll' sections of mucosa had been taken allowing the examination of long strips of mucosa up to $10.2 \mathrm{~cm}(4 \mathrm{in}$.) in length. Results were expressed as the percentage of dysplastic mucosa per slide, and for each patient the total percentage of dysplastic mucosa was expressed as a percentage of the total mucosa examined. All slides were examined at least twice on different occasions and the mean of each reading taken. Additional readings were taken only where these varied by more than $10 \%$ or where there was doubt when borderline dysplasia was present. In patients in whom a rectal carcinoma (26) was present, the proportion of frankly carcinomatous mucosa was also measured.

\section{Results}

The distribution of carcinomas in patients with ulcerative colitis is shown in Table 1. A total of 111 carcinomas in 73 patients are included with a female/male ratio of $1 \cdot 43: 1$. From this Table it is apparent that the most common site for carcinoma to develop is in the rectum, and 46/111 carcinomas $(41.5 \%)$ were at this site. Of more relevance to this study, the rectum was the site of $32 / 55$ single carcinomas $(58.2 \%)$, while $11 / 18$ patients with multiple tumours had at least one in the rectum $(61 \cdot 1 \%)$. In all, $43 / 73$ patients with carcinoma in ulcerative colitis had a carcinoma in the rectum $(58.9 \%)$. Multiple tumours were present in $9 / 30$ males $(30.0 \%)$, and $9 / 43$ females $(20.9 \%)$, an overall incidence of $24.7 \%$.

Table 2 shows the distribution of single and multiple carcinomas according to the sex of the patient. The distribution of multiple tumours is similar in both sexes with a rather more regular distribution throughout the colon when compared with non-colitic population. The distribution of 
Table 1 Site and sex of 111 carcinomas arising in ulcerative colitis in 73 patients

\begin{tabular}{lcccc}
\hline & $\begin{array}{l}\text { Male } \\
\text { (30 patients) }\end{array}$ & $\begin{array}{l}\text { Female } \\
(43 \text { patients })\end{array}$ & Total & $\%$ of total \\
\hline Caecum & 4 & 2 & 6 & $5 \cdot 4$ \\
Ascending colon & 2 & 1 & 3 & $2 \cdot 7$ \\
Hepatic flexure & 0 & 5 & 5 & $4 \cdot 5$ \\
Transverse colon & 6 & 13 & 19 & $17 \cdot 1$ \\
Splenic flexure & 3 & 7 & 10 & $9 \cdot 0$ \\
Descending colon & 6 & 6 & 12 & $10 \cdot 8$ \\
Sigmoid colon & 3 & 7 & 10 & $9 \cdot 0$ \\
Rectum & 22 & 24 & 46 & $41 \cdot 5$ \\
Total & 46 & 65 & 111 & $100 \cdot 0$ \\
\hline
\end{tabular}

Table 2 Comparison between males and females of distribution of single and multiple tumours

\begin{tabular}{llllll}
\hline & \multicolumn{2}{l}{ Multiple } & tumours & & \multicolumn{2}{l}{ Single tumours } \\
\cline { 2 - 3 } \cline { 5 - 6 } \cline { 5 - 6 } & $\begin{array}{lllll}\text { Male } \\
(8\end{array}$ & $\begin{array}{l}\text { Female } \\
(9\end{array}$ & & $\begin{array}{l}\text { Male } \\
(21\end{array}$ & $\begin{array}{l}\text { Female } \\
(34\end{array}$ \\
& patients $)$ & patients $)$ & & patients $)$ & patients $)$ \\
\hline Caecum & 3 & 1 & & 1 & 1 \\
Ascending colon & 1 & 0 & & 1 & 1 \\
Hepatic flexure & 0 & 4 & & 0 & 1 \\
Transverse colon & 6 & 6 & & 0 & 7 \\
Splenic flexure & 2 & 4 & & 1 & 3 \\
Descending colon & 5 & 2 & & 1 & 4 \\
Sigmoid colon & 2 & 6 & & 1 & 1 \\
Rectum & 6 & 8 & & 16 & 16 \\
Total & 25 & 31 & 21 & 34 \\
\hline
\end{tabular}

single tumours showed some variation between the sexes, with a greater proportion of rectal carcinomas in males $(16 / 21$ or $76 \%)$, when compared with females $(16 / 34$ or $47 \%)$.

The differing combinations of multiple carcinomas are shown in Table 3. The most frequent combination of multiple carcinomas was the expected one of transverse colon and rectum (four), there being no particular pattern in the remaining tumours other than their tendency to be distributed more evenly throughout the colon. The average number of carcinomas in this group was $3 \cdot 1$, and the highest number in any patient was eight.

The proportion of rectal mucosa showing dysplasia is shown in Table 4.

Table 5 shows the proportion of dysplastic mucosa subdivided into percentage categories between none and $100 \%$ in $25 \%$ steps. There is marked variability in the proportion of dysplastic mucosa within each category, even in those with rectal cancers. It is apparent that, in patients with carcinoma of the colon, $2 / 15$ patients $(13.3 \%)$ had no dysplasia in the slides examined, while, in another two patients, less than half of the rectal mucosa was affected. In the remaining $11(75.5 \%)$ more than half of the rectal mucosa examined was dysplastic.

In the group with dysplasia only $1 / 22$ patients
Table 3 Distribution of multiple carcinomas. Number and site of 18 patients with multiple carcinomas

\begin{tabular}{|c|c|c|c|c|c|c|c|c|c|}
\hline & & $x a$ & nd $r$ & numbe & of of tum & our & & & \\
\hline & $\begin{array}{l}M \\
2\end{array}$ & $\begin{array}{l}M \\
4\end{array}$ & $\begin{array}{l}M \\
2\end{array}$ & $\begin{array}{l}F M \\
83\end{array}$ & $\begin{array}{l}F F M \\
3554\end{array}$ & $\begin{array}{l}M \\
3\end{array}$ & $\begin{array}{l}M \\
3\end{array}$ & $\begin{array}{l}M \\
2\end{array}$ & 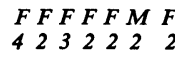 \\
\hline $\begin{array}{l}\text { Caecum } \\
\text { Ascending } \\
\text { colon }\end{array}$ & 1 & 1 & 1 & 1 & & & & & \\
\hline Hepatic flexure & & & & 2 & 11 & & & & \\
\hline $\begin{array}{l}\text { Transverse colon } \\
\text { Splenic flexure }\end{array}$ & & $\begin{array}{l}1 \\
1\end{array}$ & & 3 & 21 & 1 & 2 & 1 & $\begin{array}{llll}2 & 1 & & \\
& & 1 & 1\end{array}$ \\
\hline $\begin{array}{l}\text { colon } \\
\text { Sigmoid colon } \\
\text { Rectum }\end{array}$ & 1 & 1 & 1 & $\begin{array}{rl}21 & 1 \\
& 1\end{array}$ & $\begin{array}{lll}1 & 2 & 1 \\
1 & & \end{array}$ & 1 & 1 & 1 & 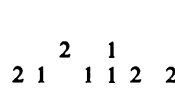 \\
\hline
\end{tabular}

M: male, F: female

Table 4 Overall proportion of rectal mucosa affected by dysplasia and carcinoma

\begin{tabular}{lllll}
\hline & $\begin{array}{l}\text { No. of } \\
\text { patients }\end{array}$ & $\begin{array}{l}\text { Avg. no. } \\
\text { slides }\end{array}$ & $\%$ dysplasia & $\%$ carcinoma \\
\hline Rectal carcinoma & 26 & $5 \cdot 2$ & 54 & 23 \\
Colon carcinoma & 15 & $2 \cdot 2$ & 69 & 0 \\
Dysplasia only & 22 & $3 \cdot 1$ & 59 & 0 \\
\hline
\end{tabular}

Table 5 Proportion of rectal mucosa affected by neoplastic transformation in 63 patients with dysplasia or carcinoma

\begin{tabular}{llllllll}
\hline & None & $1-25 \%$ & $26-50 \%$ & $51-75 \%$ & $76-99 \%$ & All & Total \\
\hline $\begin{array}{l}\text { Rectal } \\
\text { carcinoma }\end{array}$ & 0 & 4 & 7 & 9 & 4 & 2 & 26 \\
$\begin{array}{c}\text { Colon } \\
\text { carcinoma }\end{array}$ & 2 & 0 & 2 & 4 & 1 & 6 & 15 \\
$\begin{array}{c}\text { Dysplasia } \\
\text { only }\end{array}$ & 1 & 4 & 2 & 7 & 5 & 3 & 22 \\
\hline
\end{tabular}

had no evidence of rectal dysplasia in the slides examined, but in another six $(27 \%)$ less than half of the mucosa examined was dysplastic. In the remaining $15(68 \cdot 2 \%)$ more than half of the rectal mucosa examined was dysplastic.

\section{Discussion}

The distribution of carcinomas in this series is unusual because of the high proportion occurring in the rectum, particularly in males with single tumours. The overall distribution is unlike that found by Edwards and Truelove (1964), who found only six of 27 colitis carcinomas in the rectum. However, Mottet (1971) combined several series and found that $45 \%$ of carcinomas occurring in ulcerative colitis did so in the rectum. The present series is therefore more in agreement with that study. Nevertheless, $60 \%$ of all patients in this study had a rectal carcinoma, a figure similar to that found in a non- 
Table 6 Comparison between the distribution of carcinomas from the Northeast Metropolitan Region 1960-72 with the present series

\begin{tabular}{lcccr}
\hline Site & NEMR 1960-72 & $\%$ & $\begin{array}{c}\text { Present } \\
\text { series }\end{array}$ & $\%$ \\
\hline Caecum & 0 & 0 & 6 & $5 \cdot 4$ \\
Ascending colon & 1 & $3 \cdot 9$ & 3 & $2 \cdot 7$ \\
Hepatic flexure & 0 & 0 & 5 & $4 \cdot 5$ \\
Transverse colon & 4 & $15 \cdot 4$ & 19 & $17 \cdot 1$ \\
Splenic flexure & 3 & $11 \cdot 5$ & 10 & $9 \cdot 0$ \\
Descending colon & 2 & $7 \cdot 7$ & 12 & $10 \cdot 8$ \\
Sigmoid colon & 5 & $19 \cdot 2$ & 10 & $9 \cdot 0$ \\
Rectum & 11 & $42 \cdot 3$ & 46 & $41 \cdot 5$ \\
\hline
\end{tabular}

colitic population. One possible reason for this could be that this is a referral centre, so that patients with distal tumours readily found by digital examination or sigmoidoscopy were referred for definitive surgery. Conversely, those with more proximal tumours, particularly those that obstructed, would most likely undergo resection at a local hospital as an emergency procedure, rather than being referred, thus artificially selecting those patients treated at this institution.

The distribution of carcinomas in ulcerative colitis in the present series was therefore compared with those arising in the remainder of the North-east Metropolitan region of London between 1960 and 1972. The figures were kindly provided by Dr Jean Ritchie and are shown in Table 6. The distribution of carcinoma in these two series is very similar, and, if anything, shows a slight tendency for even more carcinomas to occur in the left side of the colon, particularly in the sigmoid colon. It is therefore reasonable to conclude that the figures in this present series are comparable with those found in the remainder of North and East London.

An additional potential for bias in this study is that the regular follow-up of patients at this institution is to a large extent dependent on regular sigmoidoscopy. It might therefore be expected that this would detect rectal carcinoma preferentially by direct examination (Lennard-Jones et al., 1974, 1977). However, in those papers no carcinoma was detected by direct examination. Conversely, it might be expected that the current series is weighted in favor of rectal tumours because patients with no rectal dysplasia on rectal biopsy but with a proximal carcinoma might have remained undetected. In Lennard-Jones' paper of 1977 , it is apparent that some patients have been submitted to colonoscopy and carcinoma found unexpectedly in two patients undergoing resection because of severe colonic, but not rectal, dysplasia. The number of colonoscopic biopsies taken was not stated, but the potential for other patients to have localised, but as yet undetected, carcinoma remains. Gratifyingly this has not occurred to date.

The relatively small number of patients with colon carcinoma included in the study of rectal mucosa is due to a variety of causes. Patients in whom palliative surgery for colon cancer was carried out had the rectum left intact. Also, before about 1965, the potential for the rectum to develop metachronous tumours was not fully appreciated, and slides from rectal mucosa were not available in either of these groups of patients.

The high proportion of patients with colon carcinoma having rectal dysplasia is almost certainly biased by follow-up of long-standing colitics at this institution using regular rectal biopsy. This will select those patients with rectal dysplasia who, if found to have a colonic carcinoma in the resected specimen, would be included in this study. Normal follow-up studies would therefore tend to select those patients with colon cancer having rectal dysplasia. This is of importance, as one of the questions that this paper seeks to answer is the usefulness of rectal biospy in patients with colon cancer. It would be expected that the figures given for patients with colon cancer in Tables 4 and 5 are disproportionately high, and would therefore be considerably lower in an unselected group.

The group of patients with dysplasia only are similarly selected because of the degree and extent of their dysplasia; it is therefore not surprising that such a large proportion of their rectal mucosa is involved.

In attempting to assess dysplasia in rectal biopsies quantitatively, an additional problem in all groups is exactly where the cut-off occurs from dysplastic to non-dysplastic mucosa, particularly when a spectrum of changes is present. Because of this difficulty some of the slides were examined on at least four occasions. The cut-off point was taken as the least degree of dysplasia considered to be undoubtedly neoplastic and from which a carcinoma could arise directly. This is subjective, and made on the grounds of previous experience. It could have been biased by the knowledge that these patients had either carcinoma or dysplasia in their large bowel resulting in an over-estimate of the amount of dysplastic mucosa present.

A further objection to this study is that in taking sections of mucosa, the pathologist inevitably selects areas that look abnormal. While it can be argued that clinicians taking biopsies do the same, nevertheless, this is not a complete counter-argument. There is little doubt that the low villous, velvety, rather verrucose macroscopic appearance of one variety of premalignant change is appreciated much more readily in the open fixed specimen that it is endoscopically. The pathologist, therefore, is at an 
advantage in detecting this type of change. A second lesion that is better detected in an opened fixed specimen is the small plaque-like carcinoma occasionally seen in colitis. In our experience this can be missed both sigmoidoscopically and colonoscopically and this has also been reported (Crowson et al., 1976). These points together may therefore slightly increase the amount of dysplasia recorded because of selection by the pathologist. However, dysplasia occurring in typically flat colitic mucosa is a matter of chance for both pathologist and endoscopist, while both are capable of detecting the rather less frequent polypoid lesions.

The number of slides examined is important in reducing the sampling error. Not surprisingly, patients with rectal carcinoma had the greatest number of slides of rectal mucosa. However, the difference between the average number of slides made of rectal mucosa when a colon carcinoma was also present $(2 \cdot 2)$, and when only dysplasia was present $(3 \cdot 1)$ can probably be explained on the grounds that more sections were taken from the tumour and its vicinity, including a full lymph node dissection, resulting in a tendency to take fewer sections from other areas of the bowel.

When multiple tumours are present, as was the case in $18 / 73$ patients in the current series $(24 \cdot 7 \%)$, the rectum was the site of the most distal tumour in $11(61 \cdot 1 \%)$. However, this has little practical value as, in $55 \%$ of these, proximal tumours were more advanced than the rectal tumour. Similarly, the excess of both multiple tumours and single rectal tumours in males is of no practical value. The site of the transverse colon as the second most frequent site of carcinoma can be used as an argument for the 'shift to the right' said to occur in colitic cancers, and may give the impression that, like the rectum, it is particularly predisposed to develop carcinoma. Indeed, the fact that 19 carcinomas in the present series arose in the transverse colon, compared with 10 in the splenic flexture, 12 in the descending colon, and 10 in the sigmoid colon, would seem to support this theory. However, when the length of the transverse colon is compared with that of these sites, it exceeds each in length by at least a factor of 2 . Centimetre for centimetre then, it would appear to have no greater tendency to undergo malignant transformation than the more distal colon.

In all three groups in Tables 4 and 5 it is apparent that rectal dysplasia is focal, even when a rectal carcinoma is present. Although in this study the potential for sigmoidoscopy and biopsy appears great, yet it is over-estimated because of the biases outlined above. However, the implications are clear. A single rectal biopsy may miss a focus of dysplasia if one is present because of the focal nature of dysplasia. This sampling error can be reduced by taking multiple biopsies, and further reduced by noting carefully their site of origin-for example, $8 \mathrm{~cm}$ posterior wall-so that, if negative, the site can be deliberately varied at subsequent visits. Conversely, if suspicious changes are found histologically that site can be deliberately rebiopsied. All biopsies should therefore be placed in separate, clearly labelled containers. Also, in this study, it is apparent that the rectal biopsies of some patients will provide insufficient or no warning of proximal carcinoma. As this study tends to overestimate the amount of rectal dysplasia, an even greater proportion of patients will fall into this group at other centres. Other means of detecting proximal dysplasia and carcinoma, such as colonoscopy should therefore be added to any long-term programme of cancerprevention.

We are grateful to Dr Jean Ritchie for providing access to her statistics for the North-east Metropolitan Region of London, to Dr D. Ransohoff at the University of Chicago for his helpful criticism, and to Mrs Barbara Carney for her secretarial assistance.

\section{References}

Cook, M. G., and Goligher, J. C. (1975). Carcinoma and epithelial dysplasia complicating ulcerative colitis. Gastroenterology, 68, 1127-1136.

Crowson, T. D., Ferrante, W. F., and Gathright, J. B. (1976). Colonoscopy: inefficacy for early carcinoma detection in patients with ulcerative colitis. Journal of the American Medical Association, 236, 2651-2652.

Edwards, F. C., and Truelove, S. C. (1964). The course and prognosis of ulcerative colitis. IV. Carcinoma of the colon. Gut, 5, 15-22.

Evans, D. J., Pollock, D. J. (1972). In-situ and invasive carcinoma of the colon in patients with ulcerative colitis. Gut, 13, 566-570.

Gewertz, B. L., Dent, T. L., and Appleman, H. D. (1976). Implications of precancerous rectal biopsy in patients with inflammatory bowel disease. Archives of Surgery, 111, 326-329.

Hulten, L. Kewenter, J., and Ahren, C. (1972). Precancer and carcinoma in chronic ulcerative colitis. A histopathological and clinical investigation. Scandinavian Journal of Gastroenterology, 7, 663-669.

Isaacson, P. (1976). Tissue demonstration of carcinoembryonic antigen (CEA) in ulcerative colitis. Gut, 17, 561-567.

Lennard-Jones, J. E., Misiewicz, J. J., Parrish, J. A., Ritchie, J. K., Swarbrick, E. T., and Williams, C. B. (1974). Prospective study of out-patients with extensive colitis. Lancet, 1, 1065-1067.

Lennard-Jones, J. E., Morson, B. C., Ritchie, J. K., Shove, D. C., and Williams, C. B. (1977). Cancer in colitis: assessment of the individual risk by clinical and histological criteria. Gastroenterology, 73, 1280-1289.

Morson, B. C., and Pang, L. S. C. (1967). Rectal biopsy as an aid to cancer control in ulcerative colitis. Gut, 8, 423434.

Mottet, N. K. (1971). Histopathologic Spectrum of Regional 
Enteritis and Ulcerative Colitis. (Major Problems in Pathology 21) p. 221. W. B. Saunders: Philadelphia.

Myrvold, H. E., Kock, N. G., and Ahren, C. (1974). Rectal biopsy and precancer in ulcerative colitis. Gut, 15, 301-304.

Riddell, R. H. (1976). The precarcinomatous phase of ulcerative colitis. Current Topics in Pathology, 63, 179-219. Yardley, J. H., and Keren, D. F. (1974). "Precancer" lesions in ulcerative colitis. A retrospective study of rectal biopsy and colectomy specimens. Cancer, 34, 835-844. 